\title{
Mechanism of Photo-Induced Liquid Crystal Alignment on a Poly(vinyl cinnamate) Thin Layer
}

\author{
Hee-Tak KIM, Jong-Woo LEE, Shi-Joon Sung, and Jung-Ki PARK ${ }^{\dagger}$ \\ Department of Chemical Engineering, \\ Korea Advanced Institute of Science and Technology, \\ 373-1 Kusung-dong, Yusung-gu, Daejon 305-701, Korea
}

(Received October 8, 1999; Accepted September 9, 2000)

\begin{abstract}
The mechanism of photo-induced liquid crystal (LC) alignment on a poly(vinyl cinnamate) (PVCi) thin layer was studied. By spectral analysis for the polarized UV spectra of the PVCi thin layer exposed to a polarized UV, dichroism of the photo-dimer and photo-isomer could be determined. To elucidate the mechanism of photo-induced LC alignment, changes in the dichroism of the photo-products and photoaligned LC were investigated with two successive irradiations of polarized UV $(250-340 \mathrm{~nm})$ with different polarization directions to the PVCi thin layer. The dichroism of the photoaligned LC was found to follow not the dichroism of the photo-isomer but that of the photo-dimer, which indicates that the anisotropic distribution of the photo-dimer is mainly responsible for uniform LC alignment.

KEY WORDS Photo-Alignment Mechanism / Liquid Crystal / Pply(vinyl cinnamate)/
\end{abstract}

The control of the molecular orientation of liquid crystal (LC) on thin layer of photo-reactive polymer has attracted considerable attention in the field of liquid crystal displays (LCD). ${ }^{1-14}$ This technique is effective not only for overcoming major drawbacks of mechanical rubbing treatment but also for photo-patterning of LC alignment with different azimuthal directors. The latter is expected to make it possible to reveal multi-domain LCD which can improve the viewing angle characteristics of twisted nematic (TN)-LCD. ${ }^{4,8}$

A thin film of poly(vinyl cinnamate) (PVCi), a representative photo-crosslinkable photoresist, provides a photo-active surface for achieving photo-control of the in-plane alignment of nematic LC with polarized UV irradiation. $^{2-7}$ Various recent works on structural changes of PVCi thin film upon exposure to polarized UV and consequential LC alignment demonstrate the principles of the photo-induced LC alignment. ${ }^{3,4,7,12}$ Structural anisotropy caused by anisotropic photoreaction is believed responsible for aligning LC in perpendicular to the polarization direction of UV. The photo-reaction of PVCi can be characterized by photo-

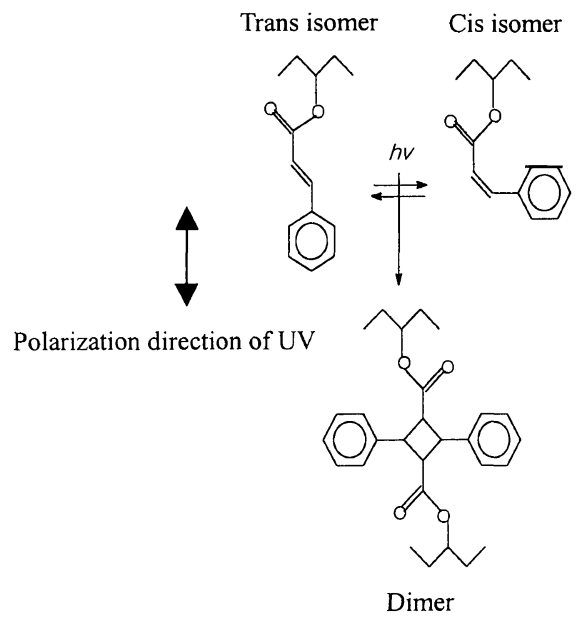

${ }^{\dagger}$ To whom all correspondence should be addressed. dimerization and photo-isomerization as described below. ${ }^{16}$

Axial-selective photo-reaction of the trans-isomer produces the cis-isomer or dimer which has orientation of the phenyl groups perpendicular to the UV polarization direction. ${ }^{3,6}$

There exists controversy on the mechanism of LC alignment on PVCi thin layer. According to Schadt and coworkers, ${ }^{3}$ surface-settled homogeneous alignment of nematic LC results from linearly polarized light induces photo-dimerization of the cinnamate moieties in PVCi thin film to form cyclobutane ring systems. Ichimura ${ }^{5,6}$ suggests that the photo-induced homogeneous LC alignment is caused by polarization photochroism at uppermost surface of the substrate resulting from repeated photo-isomerization of the cinnamate unit.

This paper reports experimental results to elucidate the mechanism of the photoalignment of LC on PVCi thin layer. Spectrophotometry at two wavelengths, one coinciding with the isosbestic point of cis-trans isomerization, makes it possible to monitor the dimerization and isomerization of PVCi simultaneously. ${ }^{12}$ Ichimura et $a l$. recently reported time-evolution of the dimer and isomer fractions during irradiation of polarized UV on PVCi thin layer. ${ }^{5,6}$ Using this spectral method, we separated the overall structural dichroism of photo-products obtained from the polarized UV spectra into two parts of the photo-dimer and photo-isomer dichroisms. By observing LC alignment together with dichroism of the photo-products, we determined the photo-product which mainly contributes to LC alignment.

\section{EXPERIMENTAL}

\section{Materials}

PVCi (weight-average molecular weight, 200000) was purchased from Aldrich and used as received. PVCi is actually a copolymer of vinyl cinnamate and vinyl alcohol. The relative mole fraction of the vinyl cinnamate unit in the copolymer determined from the ${ }^{1} \mathrm{H}$ NMR spectrum of PVCi was $76 \mathrm{~mol} \%$. The nematic liquid crys- 
tal, E7, was purchased from Merck.

\section{Film Preparation}

A 1 wt\% $N$-Methyl-2-Pyrrolidone (NMP) solution of PVCi was spin-coated onto the glass substrate at 1800 $\mathrm{rpm}$ and the cast was baked at $180^{\circ} \mathrm{C}$ to remove residual NMP. Photo-reaction of the thin layer of the polymer was carried out by irradiating polarized UV on the layer at normal incidence. Polarized UV light $(250-340 \mathrm{~nm})$ was obtained by passing the light from a $300 \mathrm{~W}$ high mercury arc (Oriel) through a UV linear dichroic polarizer (27320, Oriel) and a UV filter (51650, Oriel) for light irradiation on the layer. Intensity of the irradiated UV measured by a UV detector (UIT-150, Ushio) was 5 $\mathrm{mWcm}^{-2}$.

\section{Spectroscopic Measurements}

UV spectra were recorded with a Hewlett Packard 8452 A spectrometer. All measurements were done with a quartz slide used as reference.

\section{RESULTS AND DISCUSSION}

To find the relationship between dichroism of the photo-products and LC alignment, a double exposure experiment was performed. In this experiment, a polarized UV $(250-340 \mathrm{~nm})$ with irradiation intensity of $5 \mathrm{~mW}$ $\mathrm{cm}^{-2}$, was first irradiated on the PVCi thin layer (thickness: $50 \mathrm{~nm}$ ) in the $x$-axis direction of the experimental coordinate for $10 \mathrm{~min}\left(t_{1}\right)$. The polarization direction was rotated by $90^{\circ}$ with respect to the polarization direction of the first exposure followed by the second exposure. The anisotropy in Figure 1 originated from the transisomer and/or the photo-dimer. Due to alternation of the UV polarization direction from $x$ to $y$ axis, timeevolution of the anisotropy during double exposure shows the pattern schematically shown in Figure 1. Anisotropy developed during the first exposure would decrease in the early stage of the second exposure and, with prolonged second exposure, angular distribution of the anisotropy may vary.

Figure 2 shows polarized UV spectra of PVCi thin layers at various second exposure times $\left(t_{2}\right)$. $x$ - and $y$ polarized UV spectra were obtained with $x$ - and $y$ polarized probing UV, respectively. The peak at $274 \mathrm{~nm}$ corresponds to absorption of the cinnamate group. After the first exposure with the $x$-polarized UV, the cinnamate absorption in the $x$-polarized UV spectrum was found lower than that in the $y$-polarized UV spectrum, indicating higher photo-reaction of the cinnamate groups aligned along the polarization direction of the first exposure (see Figure 2a). With increasing irradiation time of the second exposure with the $y$-polarized UV, spectral difference between the $x$ - and $y$-polarized spectra was reduced and eventually cinnamate absorption in the $x$-polarized spectra exceeded that of the $y$ polarized spectra, which is in good agreement with the expected changes of the structural anisotropy shown in Figure 1.

The fractions of photo-dimers and photo-isomers were determined from polarized UV spectra using the following equations:

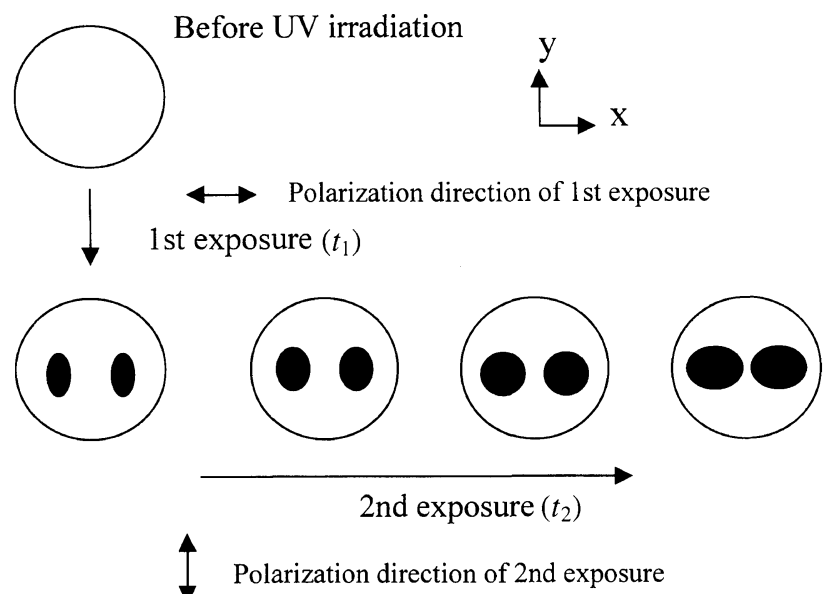

Figure 1. Schematic illustration of the double exposure experiment and development of anisotropy in the PVCi alignment layer.

$$
\begin{aligned}
& f_{\text {trans }}=\varepsilon_{\mathrm{t}} /\left(\varepsilon_{\mathrm{t}}-\varepsilon_{\mathrm{c}}\right)\left[\mathrm{A}_{274} / \mathrm{A}_{274}^{\circ}-\varepsilon_{\mathrm{t}} / \varepsilon_{\mathrm{c}}\left(\mathrm{A}_{252} / \mathrm{A}_{252}^{\circ}\right)\right] \\
& f_{\text {cis }}=\varepsilon_{\mathrm{t}} /\left(\varepsilon_{\mathrm{t}}-\varepsilon_{\mathrm{c}}\right)\left[\mathrm{A}_{252} / \mathrm{A}_{252}^{\circ}-\mathrm{A}_{274} / \mathrm{A}_{274}^{\circ}\right] \\
& f_{\text {dimer }}=1-\mathrm{A}_{252} / \mathrm{A}_{252}^{\circ}
\end{aligned}
$$

where $f_{\text {trans }}, f_{\text {cis }}$, and $f_{\text {dimer }}$ represent relative fractions of the trans-isomer, cis-isomer, and photo-dimer, $\varepsilon_{\mathrm{t}}$ and $\varepsilon_{\mathrm{c}}$ are molar extinction coefficients of the trans and cis isomers at $274 \mathrm{~nm}$. We used reported $\varepsilon_{\mathrm{t}}$ and $\varepsilon_{\mathrm{c}}$ for PVCi by Ichimura et al. ${ }^{5} \mathrm{~A}_{252}$ and $\mathrm{A}_{274}$ are absorbance at the isosbestic point ( $252 \mathrm{~nm}$ ) and absorption maximum $(274 \mathrm{~nm})$ respectively. $\mathrm{A}_{252}^{\circ}$ and $\mathrm{A}_{274}^{\circ}$ correspond to the initial absorbance of the PVCi thin layer at 252 and $274 \mathrm{~nm}$ measured before polarized UV irradiation.

The dichoric ratio (DR) for the photo-products, which represents the anisotropic distribution of the photoproduct, is defined as follows:

$$
\begin{aligned}
& \mathrm{DR}_{\text {trans }}=\left[\left(f_{\text {trans }}\right)_{x}-\left(f_{\text {trans }}\right)_{y}\right] /\left[\left(f_{\text {trans }}\right)_{x}+\left(f_{\text {trans }}\right)_{y}\right] \\
& \mathrm{DR}_{\text {cis }}=\left[\left(f_{\text {cis }}\right)_{x}-\left(f_{\text {cis }}\right)_{y}\right] /\left[\left(f_{\text {cis }}\right)_{x}+\left(f_{\text {cis }}\right)_{y}\right] \\
& \mathrm{DR}_{\text {dimer }}=\left[\left(f_{\text {dimer }}\right)_{x}-\left(f_{\text {dimer }}\right)_{y}\right] /\left[\left(f_{\text {dimer }}\right)_{x}+\left(f_{\text {dimer }}\right)_{y}\right]
\end{aligned}
$$

Subscripts $x$ and $y$ indicate UV polarization directions to obtain polarized spectra. Positive DR means higher population of a photo-product in the $x$-direction.

Figure 3 shows change of DR with irradiation time for the photo-dimer and cis-isomers during the second exposure. After the first exposure, DR for the dimers and cisisomers were negative due to generation of cis-isomers and dimers oriented in the $y$-direction by preferential photo-reaction of the trans-isomer aligned along the $x$ direction. The dichroism of the cis-isomer abruptly changed with rotation of the polarization direction. However, dichroism of the photo-dimer rather gradually varied. DR of the photo-dimer changed to positive after around $2 \mathrm{~min}$ of the second exposure.

The alignment direction of nematic LC (E7, Merck) on PVCi thin film was determined from anisotropic absorption of a small amount of dichroic dye (disperse 14) in the LC layer of the homogeneously assembled LC cell prepared with the UV-treated PVCi alignment layer. ${ }^{15}$ 


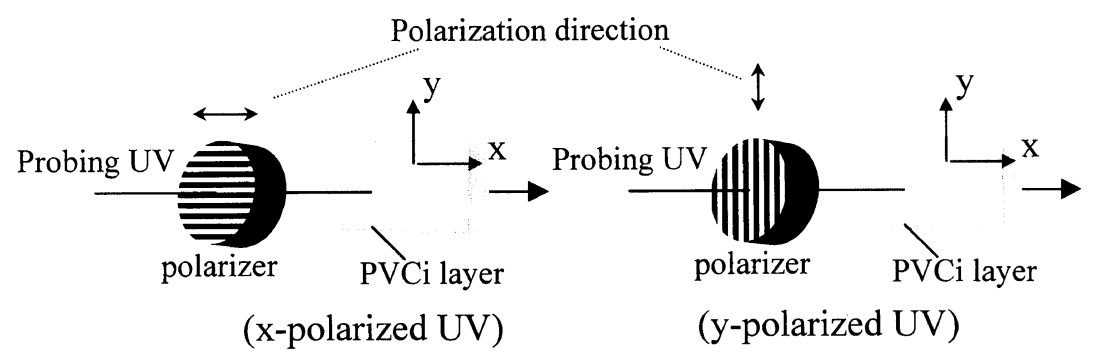

(a) $t_{1}=10 \mathrm{~min}, t_{2}=0 \mathrm{~min}$

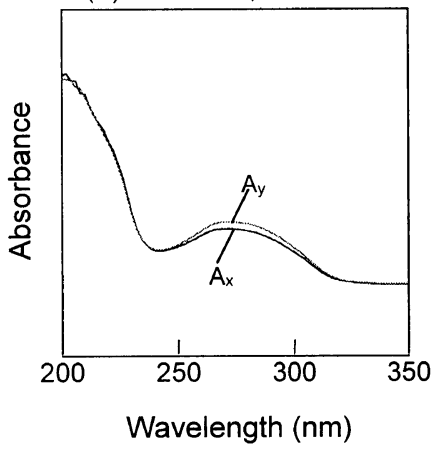

(c) $t_{1}=10 \mathrm{~min}, t_{2}=2 \mathrm{~min}$

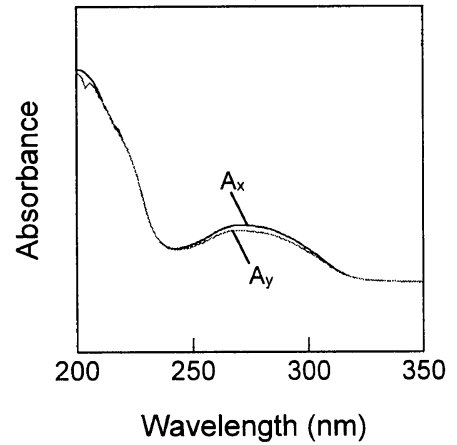

(b) $t_{1}=10 \mathrm{~min}, t_{2}=1 \mathrm{~min}$

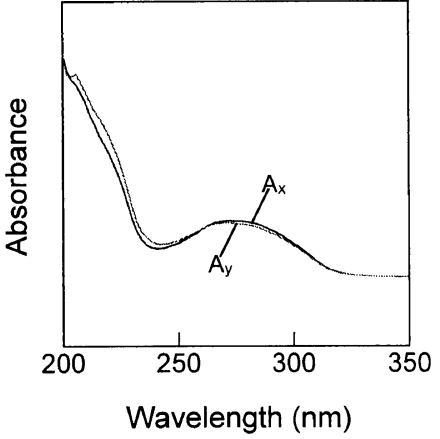

(d) $t_{1}=10 \mathrm{~min}, \mathrm{t}_{2}=3 \mathrm{~min}$

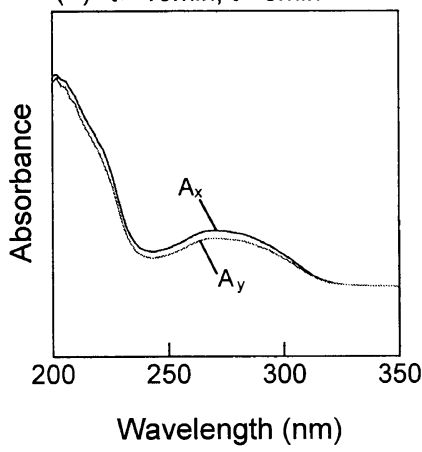

Figure 2. Polarized UV spectra of the PVCi thin layer at various second exposure times (First exposure time $\left(t_{1}\right)=10$ min, second exposure time $\left(t_{2}\right)=$ (a) $0 \mathrm{~min}$, (b) $1 \mathrm{~min}$, (c) $2 \mathrm{~min}$, (d) $3 \mathrm{~min}$ ).

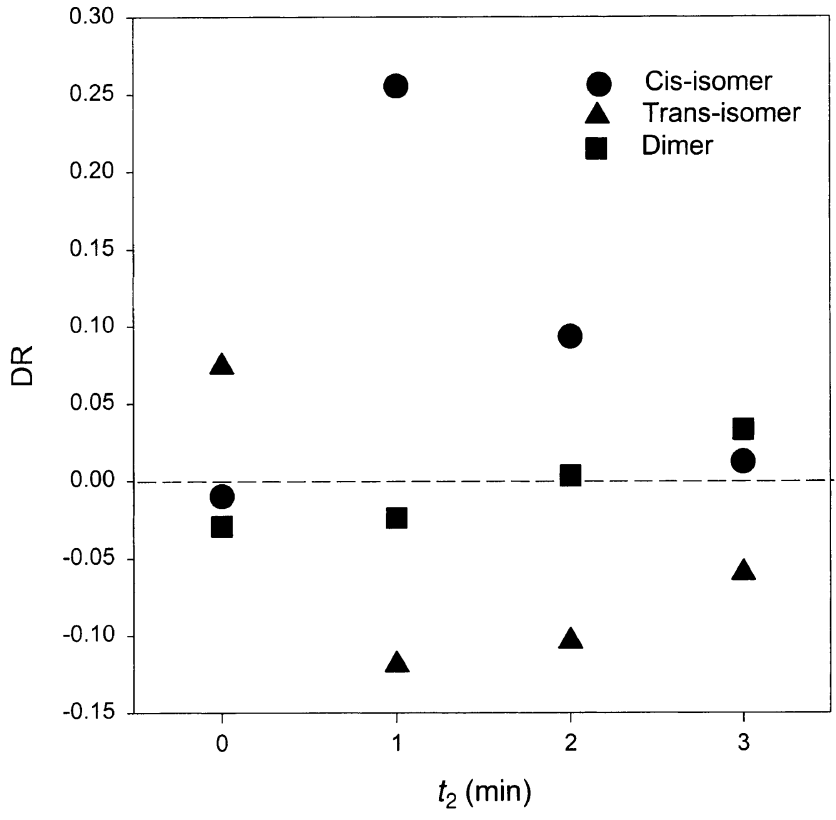

Figure 3. Plots of the dichroic ratio of the dimer, cis-isomer and trans-isomer as a function of the second exposure time.
The dichroic dye showed strong absorption at $656 \mathrm{~nm}$. The order parameter $(S)$ of LC (E7), which represents the dichroism of the photoaligned LC, was determined from,

$$
S=\left(A_{x}-A_{y}\right) /\left(A_{x}+2 A_{y}\right)
$$

where $A_{x}$ and $A_{y}$ are anisotropic absorption of the dichroic dye in the $x$-polarized and $y$-polarized UV spectra of the LC cell respectively. Negative $S$ indicates that anisotropy that developed during the first exposure still dominates the LC alignment, while positive $S$ means LC alignment is altered by the second exposure. Figure 4 shows plots of the order parameter of LC as a function of the second exposure time. Change of LC alignment direction to the $y$ direction eventually occurs at $2 \mathrm{~min}$ of the second exposure. A comparison in Figure 3 and 4 shows change of the dichroism of the photoaligned LC to resemble that of the dichroism of the photo-dimer. This strongly suggests that not the photo-isomer but the photo-dimer is mainly responsible for the LC alignment. Photo-isomerization plays a major role in the LC align- 


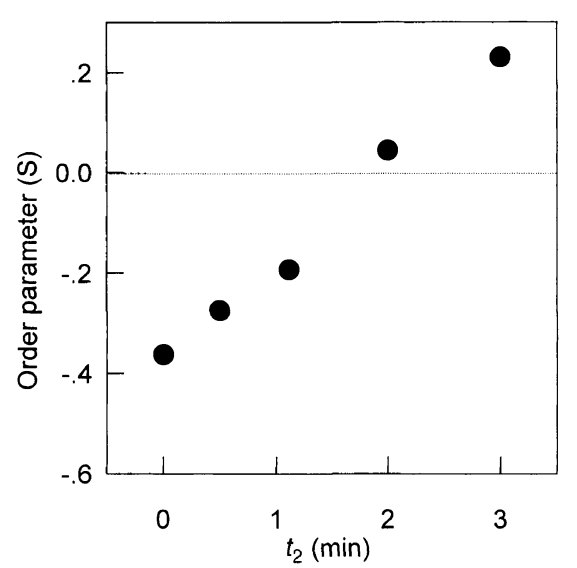

Figure 4. Plots of the dichroic ratio of the photoaligned LC as a function of the second exposure time.

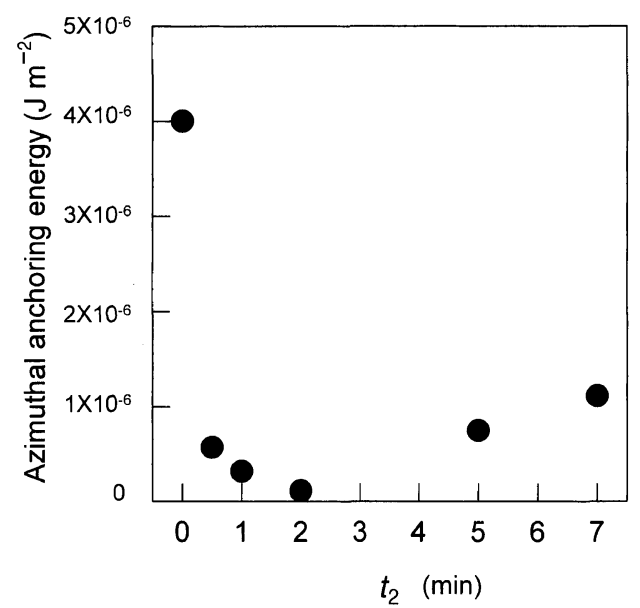

Figure 5. Plots of the azimuthal anchoring energy of the photoaligned $\mathrm{LC}$ as a function of the second exposure time.

ment, the LC alignment direction should change at 1 min of the second exposure when the change of the angluar distributon of the cis-isomer has already occurred.

Figure 5 shows change of azimuthal anchoring energy of the LC with irradiation time during the second exposure. The azimuthal anchoring energy was determined from the width of the disclination wall called the Neel wall observed from the homogeneously aligned LC cell. Detailed experimental procedures are described elesewhere. ${ }^{4,17}$ Azimuthal anchoring energy decreased at the early stage of the second exposure and after reaching a minimum, it increased with irradiation time. Minimum azimuthal anchoring energy and change of the signs of $\mathrm{DR}_{\text {dimer }}$ were found at the same irradiation time, which reconfirms the major role of the photo-dimer in the LC alignment. A relatively small second exposure compared to the first caused significant reduction of the azimuthal anchoring energy of the photoaligned LC. The higher population of unreacted trans-isomer oriented in the $y$ direction resulted by the first exposure, and thus the reaction rate of the trans-isomer in the $y$-direction during the second exposure may be much higher than that in the $x$-direction, which results in the fast diminution of dichroism during the second exposure. Larger anchoring energy after the first exposure may arise from remaining trans-isomer and dimer, while gradual increase at the second exposure after 2 min comes from the subsequent accumulation of photodimers which provide weak anchoring. This indicates that the remaining transisomer is important for achieving high anchoring energy, being in line with the mechanism given by Ichimura.

Acknowledgment. This work was supported by the Center for Advanced Functional Polymers at KAIST, KOREA in 1998.

\section{REFERENCES}

1. W. Gibbon, P. J. Shannon, S. T. Sun, and B. J. Swelin, Nature, 351, 49 (1991).

2. K. Ichimura, H. Akiyama, K. Kudo, N. Ishizuki, and S. Yamamura, Liq. Cryst., 20, 923 (1996).

3. M. Schadt, K. Schmitt, V. Kozinkov, and V. Chigrinov., Jpn. J. Appl. Phys., 31, 2155 (1992).

4. Y. Imura, S. Kobayashi, T. Hashimoto, T. Sugiyama, and K. Katoh, IEICE Trans. Elect., E79-C, 1040 (1996).

5. K. Ichimura, Y. Akita, H. Akiyama, K. Kudo, and Y. Hayashi, Macromolecules, 30, 903 (1997).

6. K. Ichimura, Y. Akita, H. Akiyama, Y. Hayashi, and K. Kudo, Jpn. J. Appl. Phys., 35, L992 (1996).

7. G. P. Bryan-Brown and I. C. Sage, Liq. Cryst., 20, 825 (1996).

8. M. Schadt, H. Seiberle, and A. Schuster, Nature, 381, 212 (1996).

9. J. Chen, D. L. Johnson, P. J. Bos, X. Wang, and J. L. West, Phys. Rev. E, 54, 2 (1996).

10. S. Song, M. Watabe, T. Adachi, T. Kobae, Y. Chen, M. Kawabata, Y. Ishida, S. Takahara, and T. Yamaoka, Jpn. J. Appl. Phys., 37, 2620 (1998).

11. H. T. Kim and J. K. Park, Jpn. J. Appl. Phys., 38, 76 (1999).

12. M. Obi, S. Morino, and K. Ichimura, Jpn. J. Appl. Phys., 38, L 145 (1999).

13. M. Obi, S. Morino, and K. Ichimura, Chem. Mater., 11, 656 (1999).

14. M. Obi, S. Morino, and K. Ichimura, Chem. Mater., 11, 1293 (1999).

15. B. Bahadur, "Liquid Crystals: Applications and Uses," World Scientific Publishing, New Jersey, NJ, 1992, vol. 3., pp 68104.

16. P. L. Egerton, E. Pitts, and A. Reiser, Macromolecules, 14, 95 (1981).

17. X. T. Li, D. H. Pei, S. Kobayashi, and Y. Iimura, Jpn. J. Appl. Phys., 36, L432 (1997). 